\title{
Parity, Relevance, and Gentle Explosiveness in the Context of Sylvan's Mate Function
}

\author{
Thomas Macaulay Ferguson \\ Cycorp and Saul Kripke Center
}

\begin{abstract}
The Routley star, an involutive function between possible worlds or set-ups against which negation is evaluated, is a hallmark feature of Richard Sylvan and Val Plumwood's set-up semantics for the logic of first-degree entailment. Less frequently acknowledged is the weaker mate function described by Sylvan and his collaborators, which results from stripping the requirement of involutivity from the Routley star. Between the mate function and the Routley star, however, lies an broad field of intermediate semantical conditions characterizing an infinite number of consequence relations closely related to first-degree entailment. In this paper, we consider the semantics and proof theory for deductive systems corresponding to set-up models in which the mate function is cyclical. We describe modifications to Anderson and Belnap's consecution calculus $L \mathrm{E}_{\mathrm{fde} 2}$ that correspond to these constraints, for which we prove soundness and completeness with respect to the set-up semantics. Finally, we show that a number of familiar metalogical properties are coordinated with the parity of a mate function's period, including refined versions of the variable-sharing property and the property of gentle explosiveness.
\end{abstract}

\section{Introduction}

The semantics for first degree entailment introduced by Richard Sylvan and Val Plumwood in [6] employs a formal device known as the Routley star, an involutive function mapping set-ups (i.e., possible-world-like states) to setups. The purpose of the device is to provide a semantics for negation tolerant of states for which a classical tautology ( $\operatorname{such}$ as $p \vee \sim p$ ) fails or within which both a formula $p$ and its negation $\sim p$ hold. Due to the involutivity of.$^{*}$, there are essentially two types of models in which the Routley star is employed: Those in which the function $*^{*}$ acts as the identity function on 
set-ups and those in which.$^{*}$ is not coextensional with the identity function. Semantically, which of these two properties is exhibited by the Routley star makes a very big difference. The operation gives rise to classical negation when it is assumed that ${ }^{*}$ is the identity function but gives rise to the weaker negation of $\mathrm{E}_{\text {fde }}$ when there may be set-ups such that $w^{*} \neq w$. Its behavior in these two cases, then, is well-understood: Semantics in which the Routley star is identity are classical, and non-classical otherwise.

It might be tempting to consider the distinction between classical and non-classical negation as corresponding to the whether or not the Routley star must map set-ups to themselves. However, corresponding (or not) to the identity function is not the only property to which we can attribute these differences.

As an example, one can argue that the properties of being the identity function or enjoying involutivity are particular cases of the property of periodicity in which a function's period is 1 and 2, respectively. One can view cyclicity as a generalized form of involutivity, insofar as there is some finite $n$ such that $n$ many applications of a function to itself is equivalent to the identity function. Framed in this manner, the distinction between the classical and paraconsistent/paracomplete negations can therefore be framed as a disagreement with respect to the parity of the period of the Routley star, i.e., we are free to consider classical negation occurring precisely when this cycle is of period 1 and attribute the behavior of the non-classical negation to the fact that the model includes cases in when the cycle is of period 2 .

Because the Routley star is defined as involutive, the investigation into this matter demands that this constraint be weakened. We therefore investigate the weaker notion of the mate function. ${ }^{M}$ described by Sylvan and his collaborators in [5, p. 326-327]. The mate function was introduced by Sylvan to serve as a relaxed or generalized version of the Routley star that, like the Routley star itself, maps set-ups to set-ups (called "mates" in this context rather than "star worlds"). Likewise, negation in this case is evaluated virtually identically, that is, by identifying the truth of a negated formula $\neg \varphi$ at a set-up $H$ with the falsity of $\varphi$ at its mate $H^{M}$. Unlike the Routley star, however, the mate function only assumes that ${ }^{M}$ is a total function choosing a mate for each set-up in a model. The mate function has been taken up anew by Gemma Robles in two recent papers [3] and [4], in which Sylvan and Bob Meyer's semantics for higher-degree relevant logics are examined in the case in which the Routley star is exchanged for its weaker cousin.

Examining the behavior of the Routley star in terms of more general patterns that emerge from the logic of the mate function allows us to frame 
the distinction between classical set-ups and non-classical set-ups as special cases of a more general distinction between the periods - or between the parity of the periods - of several mate functions. This suggests that a more general investigation of cyclical mate functions has merit. In what follows, then, we study the logic of first-degree models with mate functions that are assumed to be cyclical functions with a finite period, with an eye towards to securing a richer understanding of negation in first-degree entailment.

\section{Formal Preliminaries}

Primarily, we will be working in the language of zeroth-degree formulae in which no instances of the intensional conditional connective $\rightarrow$ appears. This language $-\mathcal{L}_{z d f}$-is recursively constructed from a denumerable set of atoms $\mathbf{A t}=\left\{p_{0}, \ldots, p_{n}, \ldots, q_{0}, \ldots\right\}$ in the following way:

Definition 1. $\mathcal{L}_{z d f}$ is defined in Backus-Naur form with $p \in$ At:

$$
\varphi::=p|\sim \varphi| \varphi \wedge \varphi \mid \varphi \vee \varphi
$$

Definition 2. $\mathcal{L}$ is defined as the set $\left\{\varphi \rightarrow \psi \mid \varphi, \psi \in \mathcal{L}_{z d f}\right\}$.

We will frequently be concerned with finite strings of negation symbols of arbitrary length. For this reason, we adopt the following convention:

Definition 3. We write " $\sim_{n} \varphi$ " as a shorthand for a string of $n$ many (possibly zero) negation signs followed by the formula $\varphi$.

Many discussions in the following subsume cases of "positive" formulaei.e., formulae in front of which no negation signs appear - as a special case of a more general treatment of formulae of the form $\sim_{n} \varphi$. For this reason, it is especially important to reinforce that $n$ in Definition 3 may be zero and, consequently, the identity between the representation of a formula as $\sim_{0} \varphi$ and its unqualified representation as $\varphi$.

\subsection{Set-Up Models}

Recall that a set-up is essentially a collection of atomic formulae. The truth of an atom $p$ in a set-up corresponds to its inclusion in the set-up qua set.

Definition 4. A first-degree model with a mate function is a triple $\mathscr{M}=$ $\left\langle G, K,{ }^{M}\right\rangle$, where $K$ is a nonempty collection of atomic set-ups, $G \in K$ is a 
distinguished set-up ${ }^{1}$ and ${ }^{M}: K \rightarrow K$ is a unary mate function such that for all $H \in K$, there exists a set-up $I \in K$ such that $H^{M}=H$.

As a matter of convention, rather than denoting the result of the result of $n$ applications of the mate function to a set-up $H$ by a lengthy superscript of $n$ many instances of . ${ }^{M}$, we instead denote this set-up by $H^{M_{n}}$. By this convention, then, one may recognize the identities $H^{M_{0}}=H$ and $H^{M_{2}}=$ $H^{M M}$.

The truth of a formula $\varphi \in \mathcal{L}_{\text {zdf }}$ or $\varphi \rightarrow \psi \in \mathcal{L}$ is determined recursively in a style that should be familiar to anyone familiar with possible worlds in logic.

Definition 5. The semantic conditions recursively determining truth in a set-up are as follows:

$$
\begin{array}{lll}
H \Vdash p & \text { iff } & p \in H \text { for } p \in \mathbf{A t} \\
H \Vdash \sim \varphi & \text { iff } & H^{M} \nVdash \varphi \\
H \Vdash \varphi \wedge \psi & \text { iff } & H \Vdash \varphi \text { and } H \Vdash \psi \\
H \Vdash \varphi \vee \psi & \text { iff } & H \Vdash \varphi \text { or } H \Vdash \psi \\
G \Vdash \varphi \rightarrow \psi & \text { iff } & \text { for all } H \text { such that } H \Vdash \varphi, \text { also } H \Vdash \psi
\end{array}
$$

Again, note that the significance of the distinguished world $G$ follows entirely from its role in evaluating first-degree formulae $\varphi \rightarrow \psi$. Because our interest in $G$ is so restricted, it matters little what value is chosen for $G^{M}$.

It is also worth noting that despite the impoverished account of negation, we nevertheless retain some form of De Morgan's laws, e.g., applying a string of an odd number of negation signs to a conjunction $\varphi \wedge \psi$ results in a formula that is evaluated disjunctively. The distinct behavior of even and odd strings of negations is apparent in the following observation: That a case like $H \Vdash \sim_{2 n}(\varphi \wedge \psi)$ holds corresponds to the truth of $\varphi \wedge \psi$ at some set-up (i.e., that $H^{M_{2 n}} \Vdash \varphi \wedge \psi$ ). In contrast, the truth of $\sim_{2 n+1}(\varphi \wedge \psi$ ) at $H$ is determined by the falsity of $\varphi \wedge \psi$ at another set-up (i.e., that $\left.H^{M_{2 n+1}} \nVdash \wedge \psi\right)$.

Before considering refinements of the models described in Definition 4 , let us stop to explicitly state one important - if relatively routine - fact about any such model.

\footnotetext{
${ }^{1} \mathrm{~A}$ referee has pointed out that the use of the $\rightarrow$ is somewhat superfluous and removing it would spare the need for a distinguished set-up. I concede the point but retain this definition for historical continuity with the presentation of Sylvan and Plumwood.
} 
Lemma 1. For all models, $H \Vdash \sim \sim \varphi$ iff $H^{M M} \Vdash \varphi$

Proof. By the semantic interpretation of $\sim$.

Having shown this, let us strengthen the model-theoretic properties of the mate function by adding the additional stipulation that ${ }^{M}$ be a cyclical function.

Definition 6. Where $n$ is a natural number, a first-degree model with a mate function (in the sense of Definition 4) is cyclical with period $n$ if the mate function. ${ }^{M}$ enjoys the additional property that:

- for all $H \in K \backslash\{G\}, H^{M_{n}}=H^{M}$

The evaluation of the truth of a formula at a set-up described in Definition 5 continues to hold with respect to these classes of set-up models.

Lemma 2. For models with periodicity n,

$$
H \Vdash \sim_{2 n} \varphi \text { iff } H \Vdash \varphi
$$

Proof. By Lemma 1, for every $H$, we can construct a finite chain of equivalences:

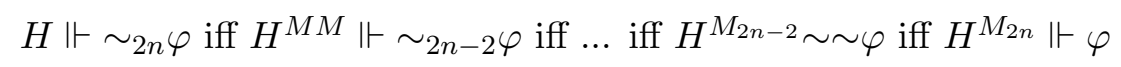

But whether the model has periodicity $n$ or periodicity $2 n, H=H^{M_{2 n}}$, concluding the proof.

Having described the intended model theory in some level of detail, we now turn to the matter of defining appropriate proof theories for these systems.

\subsection{Consecution Calculi for Cyclic $E_{f d e}$}

To provide proof theory adequate for the models described in the previous section, we take as our point of departure the consecution calculus $L \mathrm{E}_{\text {fde } 2}$ described in Anderson and Belnap [1, p. 179], making slight modifications as needed. We first introduce a very weak calculus $L \mathrm{E}_{\text {fde2 }}^{\llbracket \omega \rrbracket}$ that serves as the basic consecution calculus for first-degree entailment with the mate function. Recall that a sequent is an object of the form $\Gamma \vdash \Delta$ where $\Gamma$ and $\Delta$ are (possibly empty) sets of formulae of $\mathcal{L}_{z d f}$. We follow the conventional abuses of notation by equating a formula with its singleton (whence $\varphi \vdash \psi$ is shorthand for $\{\varphi\} \vdash\{\psi\}$ ) and equating commas with set union (whence $\Gamma_{0}, \Gamma_{1} \vdash \Delta_{0}, \Delta_{1}$ is identified with $\left.\Gamma_{0} \cup \Gamma_{1} \vdash \Delta_{0} \cup \Delta_{1}\right)$. We define the basic calculus as follows: 
Definition 7. The system $L \mathrm{E}_{\mathrm{fde} 2}^{\llbracket \omega \rrbracket}$ is a consecution calculus including axioms of the form:

$$
\overline{\Gamma_{0}, \varphi, \Gamma_{1} \vdash \Delta_{0}, \varphi, \Delta_{1}}[\text { Axiom }]
$$

the following even inference rules for even natural numbers $k$ (note that it is possible that $k=0$ )

$$
\begin{gathered}
\frac{\Gamma_{0}, \sim_{k} \varphi, \sim_{k} \psi, \Gamma_{1} \vdash \Delta}{\Gamma_{0}, \sim_{k}(\varphi \wedge \psi), \Gamma_{1} \vdash \Delta}\left[\wedge_{\mathrm{e}} L\right] \\
\frac{\Gamma \vdash \Delta_{0}, \sim_{k} \varphi, \Delta_{1} \quad \Gamma \vdash \Delta_{0}, \sim_{k} \psi, \Delta_{1}}{\Gamma \vdash \Delta_{0}, \sim_{k}(\varphi \wedge \psi), \Delta_{1}}\left[\wedge_{\mathrm{e}} R\right] \\
\frac{\Gamma_{0}, \sim_{k} \varphi, \Gamma_{1} \vdash \Delta \quad \Gamma_{0}, \sim_{k} \psi, \Gamma_{1} \vdash \Delta}{\Gamma_{0}, \sim_{k}(\varphi \vee \psi), \Gamma_{1} \vdash \Delta}\left[\vee_{\mathrm{e}} L\right] \\
\frac{\Gamma \vdash \Delta_{0}, \sim_{k} \varphi, \sim_{k} \psi, \Delta_{1}}{\Gamma \vdash \Delta_{0}, \sim_{k}(\varphi \vee \psi), \Delta_{1}}\left[\vee_{\mathrm{e}} R\right]
\end{gathered}
$$

in conjunction with odd inference rules for odd natural numbers $k$ :

$$
\begin{gathered}
\frac{\Gamma_{0}, \sim_{k} \varphi, \Gamma_{1} \vdash \Delta \quad \Gamma_{0}, \sim_{k} \psi, \Gamma_{1} \vdash \Delta}{\Gamma_{0}, \sim_{k}(\varphi \wedge \psi), \Gamma_{1} \vdash \Delta}\left[\wedge_{\circ} L\right] \\
\frac{\Gamma \vdash \Delta_{0}, \sim_{k} \varphi, \sim_{k} \psi, \Delta_{1}}{\Gamma \vdash \Delta_{0}, \sim_{k}(\varphi \wedge \psi), \Delta_{1}}\left[\wedge_{\circ} R\right] \\
\frac{\Gamma_{0}, \sim_{k} \varphi, \sim_{k} \psi, \Gamma_{1} \vdash \Delta}{\Gamma_{0}, \sim_{k}(\varphi \vee \psi), \Gamma_{1} \vdash \Delta}\left[\vee_{\circ} L\right] \\
\frac{\Gamma \vdash \Delta_{0}, \sim_{k} \varphi, \Delta_{1} \quad \Gamma \vdash \Delta_{0}, \sim_{k} \psi, \Delta_{1}}{\Gamma \vdash \Delta_{0}, \sim_{k}(\varphi \vee \psi), \Delta_{1}}\left[\vee_{\circ} R\right]
\end{gathered}
$$

Finally, derivability of a first degree formula $\varphi \rightarrow \psi$ corresponds to the derivability of the sequent $\varphi \vdash \psi$.

Examination of the foregoing calculus reveals that $L \mathrm{E}_{\text {fde2 }}^{\llbracket \omega \rrbracket}$ is almost entirely agnostic concerning the interpretation of negation in first-degree entailment, capable of making only the distinction that strings of even numbers of negation signs and strings of odd numbers of negation signs are dual to one another with respect to how they commute through conjunction and disjunction. Against the foregoing rules, the formula $\sim_{m} p$ is, at first blush, wholly independent from the formula $\sim_{n} p$ whenever $m$ and $n$ are distinct. 
The series of consecution calculi described in the next few pages are distinguished with respect to the periodicity implicit in their rules for negation. Now, to refine the goal of studying systems with cyclical mate functions, a deeper goal is to study the logical properties following from the parity of the period of a mate function. In the proof-theoretic case, one such feature of parity is reflected in the need to maintain two distinct schemes by which $L E_{f d e 2}^{\llbracket \omega \rrbracket}$ is to be extended. We then provide two schemes for defining systems $L \mathrm{E}_{\mathrm{fde} 2}^{\llbracket n \rrbracket}$-one scheme for the case in which $n$ is even and another for the case in which $n$ is odd. First, we consider the even case:

Definition 8. For an even natural number $2 n$, the system $L \mathrm{E}_{\text {fde2 }}^{\llbracket 2 n \rrbracket}$ is defined by enriching the calculus $L \mathrm{E}_{\mathrm{fde} 2}^{\llbracket \omega \rrbracket}$ with the following pair of inferences for negation:

$$
\frac{\Gamma_{0}, \varphi, \Gamma_{1} \vdash \Delta}{\Gamma_{0}, \sim_{2 n} \varphi, \Gamma_{1} \vdash \Delta}\left[\sim_{2 n} L\right] \quad \frac{\Gamma \vdash \Delta_{0}, \varphi, \Delta_{1}}{\Gamma \vdash \Delta_{0}, \sim_{2 n} \varphi, \Delta_{1}}\left[\sim_{2 n} R\right]
$$

For a natural number $2 n$, the corresponding semantic constraint on the mate function ${ }^{M}$ entails that the iteration of $2 n$ many applications of ${ }^{M}$ is equivalent to the identity function. This feature is captured by the rules $\left[\sim_{2 n} L\right]$ and $\left[\sim_{2 n} R\right]$ by which we may introduce negation signs into a proof. In a system $L \mathrm{E}_{\text {fde } 2}^{\llbracket 2 n \rrbracket}$, to demonstrate the equivalence between $\sim_{2 n} \varphi$ and $\varphi$ is task made simple by the ease by which one may construct proofs of the first-degree formulae $\sim_{2 n} \varphi \rightarrow \varphi$ and $\varphi \rightarrow \sim_{2 n} \varphi$.

It is also worthwhile to note that reviewing the rules of Anderson and Belnap's consecution calculus $L \mathrm{E}_{\mathrm{fde} 2}$ quickly shows it to share its collection of derivable sequents $L \mathrm{E}_{\mathrm{fde} 2}^{\llbracket 2 \rrbracket}$, entailing that this calculus corresponds to firstdegree entailment. If one considers that the involutive Routley star is $a u$ fond a mate function with period 2 and one recalls that Anderson and Belnap's calculus is sound and complete for Sylvan and Plumwood's firstdegree models with the Routley star, then the equivalence between the two calculi becomes entirely natural.

Now, as suggested earlier, the parity of the odd case radically impacts the behavior of consequence in systems $L \mathrm{E}_{\text {fde2 } 2}^{\llbracket 2 n+1 \rrbracket}$, introducing features not present in any of the even cases. The divide between systems with even and odd parity can now be brought into focus as we schematically define the corresponding consecution calculi by making the following augmentations to $L \mathrm{E}_{\mathrm{fde} 2}^{\llbracket \omega \rrbracket}$ :

Australasian Journal of Logic (15:2) 2018 Article no. 3.7 
Definition 9. For an odd natural number $2 n+1$, the consecution calculust $L \mathrm{E}_{\mathrm{fde} 2}^{\llbracket 2 n+1 \rrbracket}$ results from adding to the consecution calculus $L \mathrm{E}_{\mathrm{fde} 2}^{\llbracket \omega \rrbracket}$ the inferences of $\left[\sim_{4 n+2} L\right]$ and $\left[\sim_{4 n+2} R\right]$ :

$$
\frac{\Gamma_{0}, \varphi, \Gamma_{1} \vdash \Delta}{\Gamma_{0}, \sim_{4 n+2} \varphi, \Gamma_{1} \vdash \Delta}\left[\sim_{4 n+2} L\right] \quad \frac{\Gamma \vdash \Delta_{0}, \varphi, \Delta_{1}}{\Gamma \vdash \Delta_{0}, \sim_{4 n+2} \varphi, \Delta_{1}}\left[\sim_{4 n+2} R\right]
$$

To these rules, we also add the following two axioms:

$$
\overline{\Gamma_{0}, \varphi, \sim_{2 n+1} \varphi, \Gamma_{1} \vdash \Delta}\left[\perp_{2 n+1} L\right] \quad \overline{\Gamma \vdash \Delta_{0}, \varphi, \sim_{2 n+1} \varphi, \Delta_{1}}\left[\top_{2 n+1} R\right]
$$

A tentative, semantic reading of these axioms suggests that $\varphi \wedge \sim_{2 n+1} \varphi$ is an absurd proposition, from which all other propositions may be derived, while formulae of the form $\varphi \vee \sim_{2 n+1} \varphi$ are tautologies that are vacuous consequences of any arbitrary proposition. Hence, the rules $\left[\perp_{2 n+1} L\right]$ and $\left[\top_{2 n+1} R\right]$ are recognizable as generalized versions of ex contradictione quodlibet and excluded middle, respectively.

Note also that these facts - again, features peculiar to the odd case- have taken us far afield from the logic of first-degree entailment, in which there exist no formulae that are capable of playing the roles of a top or bottom constant. In other words, in first-degree entailment, there exist neither tautologies nor logical absurdities. That such notions are definable precisely in systems in which the mate function has odd parity is quite interesting.

Now, we wish to prove some meta-properties of these systems that capture various aspects of the interrelationships that emerge between instances of the aforementioned calculi.

Observation 1. Suppose that the rules $\left[\sim_{k} L\right]$ and $\left[\sim_{k} R\right]$ are admissible in a calculus $L \mathrm{E}_{\mathrm{fde} 2}^{\llbracket n \rrbracket}$. Then for all natural numbers $j$, the rules $\left[\sim_{j \cdot k} L\right]$ and $\left[\sim_{j \cdot k} R\right]$ are also admissible in $L \mathrm{E}_{\mathrm{fde} 2}^{\llbracket n \rrbracket}$.

Proof. This follows from the rules governing negation. Without loss of generality, consider $\left[\sim_{j \cdot k} L\right]$. Then any instance of the rule-which introduces a string $\sim_{j \cdot k}$ to a formula $\varphi$-may be emulated by either $j$ many successive applications of $\left[\sim_{k} L\right]$ or $k$ many successive applications of $\left[\sim_{j} L\right]$ to the formula $\varphi$. 
Now, for two calculi $S$ and $T$ in the above family, write $S \subseteq T$ if every rule in $S$ is admissible in $T$. With respect to the calculi introduced here, it follows that whenever $S \subseteq T$, anything provable in $S$ is provable in $T$.

Then we may describe a complete characterization of the conditions under which one system $L \mathrm{E}_{\text {fde2 }}^{\llbracket n \rrbracket}$ is a subsystem of a second system $L \mathrm{E}_{\text {fde2 }}^{\llbracket n \rrbracket}$ :

Observation 2. $L \mathrm{E}_{\text {fde } 2}^{\llbracket n \rrbracket} \subseteq L \mathrm{E}_{\text {fde2 }}^{\llbracket m \rrbracket}$ iff $m \mid n$.

Proof. For right-to-left, we consider each of the two cases:

- If $n$ is odd, then either $m=n$ or not. If $m=n$, then the left-hand side holds trivially, so suppose that $m \neq n$. Note that in this case, calculation establishes that $2 m \mid(n-m)$. Now, because $m \mid n, m$ must be odd, whence $\left[\sim_{2 m} L\right],\left[\sim_{2 m} R\right],\left[\perp_{m} L\right]$ and $\left[\top_{m} R\right]$ hold for $L \mathrm{E}_{\text {fde2 }}^{\llbracket m \rrbracket}$. Then for any $L \mathrm{E}_{\text {fde2 }}^{\llbracket n \rrbracket}$ proof, applications of $\left[\sim_{2 n} L\right]$ and $\left[\sim_{2 n} R\right]$ can be emulated by the application of $\frac{n}{m}$ many applications of $\left[\sim_{2 m} L\right]$ or $\left[\sim_{2 m} R\right]$, respectively. Furthermore, any instance of axioms $\left[\perp_{n} L\right]$ or $\left[\top_{n} R\right]$ can be emulated by an application of $\left[\perp_{m} L\right]$ (respectively, $\left[\top_{m} R\right]$ ) followed by $\frac{n-m}{2 m}$ many applications of $\left[\sim_{2 m} L\right]$ (respectively, $\left[\sim_{2 m} R\right]$ ). Because $2 m \mid(n-m)$, this will be an admissible series of rules.

- In the second case, there are two subcases: one in which $m$ is even and one in which $m$ is odd. In the first subcase in which both $m$ and $n$ are even, any instance of $\left[\sim_{n} L\right]$ (respectively, $\left[\sim_{n} R\right]$ ) can be emulated by $\frac{n}{m}$ many applications of $\left[\sim_{m} L\right]$ (respectively, $\left[\sim_{m} R\right]$ ). In the case in which $n$ is even but $m$ is odd, then it follows that $2 m \mid n$. Now, because $L \mathrm{E}_{\text {fde2 }}^{\llbracket m \rrbracket}$ has rules $\left[\sim_{2 m} L\right]$ and $\left[\sim_{2 m} R\right]$, then any instance of $\left[\sim_{n} L\right]$ can be emulated by $\frac{n}{2 m}$ many instances of $\left[\sim_{2 m} L\right]$ (and mutatis mutandis for $\left[\sim_{n} R\right]$ ).

For left-to-right, we establish that $m \mid n$. To show this, suppose $L \mathrm{E}_{\text {fde2 }}^{\llbracket n \rrbracket} \subseteq$ $L \mathrm{E}_{\text {fde2 } 2}^{\llbracket m \rrbracket}$. If $n$ is odd, then $L \mathrm{E}_{\text {fde2 }}^{\llbracket n \rrbracket}$ proves $p \wedge \sim_{n} p \rightarrow q$ and, because this cannot be proven in a system with even parity $\int^{2} m$ must, too, be odd. In this case, $L \mathrm{E}_{\text {fde2 }}^{\llbracket m \rrbracket}$ must be able to prove the $L \mathrm{E}_{\text {fde2 }}^{\llbracket n \rrbracket}$ theorem $\sim_{2 n} p \rightarrow p$. Because the consecution calculus has the subformula property, any emulation of this will have to come from applications of some number of applications of a rule $\left[\sim_{2 j} L\right]$ that cumulatively amount to an application of $\left[\sim_{2 n} L\right]$. Since the only left negation introduction rule available to $L \mathrm{E}_{\text {fde2 }}^{\llbracket m \rrbracket}$ is $\left[\sim_{2 m} L\right]$, this will be admissible in $L \mathrm{E}_{\mathrm{fde} 2}^{\llbracket m \rrbracket}$ only if the system can employ $k$ many applications of $\left[\sim_{2 m} L\right]$ where $k \cdot m=n$. But in this case, $k=\frac{n}{m}$, entailing that $m \mid n$.

\footnotetext{
${ }^{2}$ For formal validation of this point, see Observation 6
} 
When $n$ is even, then the $L \mathrm{E}_{\text {fde2 }}^{\llbracket n \rrbracket}$ theorem $\sim_{n} p \rightarrow p$ can only be proven in the system $L \mathrm{E}_{\text {fde2 }}^{\llbracket m \rrbracket}$ if it can give a sequence of $j$ many applications of $\left[\sim_{m} L\right]$ (when $m$ is even) or $k$ many applications of $\left[\sim_{2 m} L\right]$ (when $m$ is odd). But in these two cases, either $j=\frac{n}{m}$ or $2 k=\frac{n}{m}$, respectively, whence $m \mid n$.

Observation 2 provides an exhaustive characterization of these systems by which, for any two systems, the question of whether one is a subsystem of the other may be swiftly solved by calculation. As an example, that the calculus $L \mathrm{E}_{\mathrm{fde} 2}^{\llbracket 21 \rrbracket}$ is a subsystem of the calculus $L \mathrm{E}_{\mathrm{fde} 2}^{\llbracket 7 \rrbracket}$ may be established by inspection of the periods.

\subsection{Soundness and Completeness}

Having carefully described the model theory and proof theory for first-degree systems with cyclical mate functions in Sections 2.1 and 2.2, we now turn our attention to establishing the important metalogical properties that hold between each of the classes of models and its corresponding consecution calculi.

First, we present a number of helpful lemmas and definitions. Say that a set of formulae $\Gamma$ of $\mathcal{L}_{\text {zdf }}$ formulae is prime if for all disjunctive formulae $\varphi \vee$ $\psi \in \Gamma$ (including conjunctions negated by an odd string $\sim_{m}$ or disjunctions negated by an even string $\sim_{n}$ ), either $\varphi \in \Gamma$ or $\psi \in \Gamma$.

Lemma 3. Suppose that $\vdash \varphi \rightarrow \psi$ is not derivable in $L \mathrm{E}_{\mathrm{fde} 2}^{\llbracket n \rrbracket}$. Then there exists a prime, non-trivial, and deductively closed set $\Gamma$ such that $\varphi \in \Gamma$ and for no $\Gamma^{\prime} \subseteq \Gamma$ is $\Gamma^{\prime} \vdash \psi$ a derivable sequent.

Proof. Because $\sim_{k}(\varphi \wedge \psi) \rightarrow\left(\sim_{k} \varphi \vee \sim_{k} \psi\right)$ and its converse are provable when $k$ is odd in $L \mathrm{E}_{\text {fde } 2}^{\llbracket \omega \rrbracket}$, we are able to treat oddly negated conjunctions as disjunctions, allowing us to infer the lemma from a standard Henkin-style argument.

A further useful tool will be the presentation, for each natural number $n$, of a normal form for zeroth-degree formulae. We present the definition of each normal form schematically, taking care to note that two normal forms $(\varphi)^{N F_{m}}$ and $(\varphi)^{N F_{n}}$ may differ when $m$ and $n$ are distinct.

Definition 10. For all $n>1$, each zeroth-degree formula $\varphi$ has a literal normal form $\varphi^{N F_{n}}$. Noting that in the below $k$ can be 0 (so that, e.g., $\sim_{0} p$ is just $p$ ), we define these translations recursively: 
- $\left(\sim_{k} p\right)^{N F_{n}}= \begin{cases}\sim_{k} p & \text { for } k<n \\ \left(\sim_{k-n} p\right)^{N F_{n}} & \text { for } k \geq n\end{cases}$

- $\sim_{2 k}(\varphi \wedge \psi)^{N F_{n}}=\left(\sim_{2 k} \varphi\right)^{N F_{n}} \wedge\left(\sim_{2 k} \psi\right)^{N F_{n}}$

- $\sim_{2 k}(\varphi \vee \psi)^{N F_{n}}=\left(\sim_{2 k} \varphi\right)^{N F_{n}} \vee\left(\sim_{2 k} \psi\right)^{N F_{n}}$

- $\sim_{2 k+1}(\varphi \wedge \psi)^{N F_{n}}=\left(\sim_{2 k+1} \varphi\right)^{N F_{n}} \vee\left(\sim_{2 k+1} \psi\right)^{N F_{n}}$

- $\sim_{2 k+1}(\varphi \vee \psi)^{N F_{n}}=\left(\sim_{2 k+1} \varphi\right)^{N F_{n}} \wedge\left(\sim_{2 k+1} \psi\right)^{N F_{n}}$

The adequacy of each normal form ${ }^{N F_{n}}$ is tied to the periodicity of the mate function (in the semantic case) or the rules for introducing negations (in the proof-theoretic case). We demonstrate the adequacy of the translation in two parts, showing first that $\varphi$ and its normal form are equivalent in appropriate models, and then showing that the two are equivalent in appropriate consecution calculi.

First, we demonstrate that for an odd number $n$, the formulae $\varphi$ and $(\varphi)^{N F_{n}}$ are model-theoretically equivalent in any model whose mate function has either period $n$ or period $2 n$.

Lemma 4. Let $n$ be odd and let $\mathfrak{M}$ be a model in which ${ }^{M}$ has either period $n$ or period $2 n$. Then for any set-up $H, H \Vdash \varphi$ if and only if $H \Vdash \varphi^{N F_{2 n}}$.

Proof. In the case in which $\varphi$ is a sequence of negation signs followed by an atom, this holds trivially. By Lemma 2, one can eliminate strings of negation symbols of length $2 n$, which mirrors the structure of the definition of normal form.

For formulae in which a binary operator appears, consider the cases in we deal with a string of negation signs of length $2 k$ (n.b. that, in subsuming $\sim_{0}$ in this case, this clause covers non-negated conjunctions and disjunctions):

- $H \Vdash \sim_{2 k}(\varphi \wedge \psi)$ iff $H^{M_{2 k}} \Vdash(\varphi \wedge \psi)$ iff $H^{M_{2 k}} \Vdash \varphi$ and $H^{M_{2 k}} \Vdash \psi$ iff $H \Vdash \sim_{2 k} \varphi$ and $H \Vdash \sim_{2 k} \psi$ iff $H \Vdash \sim_{2 k} \varphi \wedge \sim_{2 k} \psi$.

- $H \Vdash \sim_{2 k}(\varphi \vee \psi)$ iff $H^{M_{2 k}} \Vdash(\varphi \vee \psi)$ iff $H^{M_{2 k}} \Vdash \varphi$ or $H^{M_{2 k}} \Vdash \psi$ iff $H \Vdash \sim_{2 k} \varphi$ or $H \Vdash \sim_{2 k} \psi$ iff $H \Vdash \sim_{2 k} \varphi \vee \sim_{2 k} \psi$.

Finally, consider the cases involving a string of an odd number of negation signs of length $2 k+1$ :

- $H \Vdash \sim_{2 k+1}(\varphi \wedge \psi)$ iff $H^{M_{2 k+1}} \nVdash(\varphi \wedge \psi)$ iff $H^{M_{2 k+1}} \nVdash \varphi$ or $H^{M_{2 k+1}} \nVdash \psi$ iff $H \Vdash \sim_{2 k+1} \varphi$ or $H \Vdash \sim_{2 k+1} \psi$ iff $H \Vdash \sim_{2 k+1} \varphi \vee \sim_{2 k+1} \psi$. 
- $H \Vdash \sim_{2 k+1}(\varphi \vee \psi)$ iff $H^{M_{2 k+1}} \nVdash(\varphi \vee \psi)$ iff $H^{M_{2 k+1}} \nVdash \varphi$ and $H^{M_{2 k+1}} \nVdash \psi$ iff $H \Vdash \sim_{2 k+1} \varphi$ and $H \Vdash \sim_{2 k+1} \psi$ iff $H \Vdash \sim_{2 k+1} \varphi \wedge \sim_{2 k+1} \psi$.

In all of these cases, we find that $H \Vdash \varphi$ if and only if $H \Vdash \varphi^{N F_{2 n}}$ as required.

With the semantic lemma in hand, we now look to proving an analogous syntactic lemma demonstrating that $\varphi$ and $(\varphi)^{N F_{n}}$ are interderivable in appropriate consecution calculi.

We say that $\Gamma$ is deductively closed modulo one of our consecution calculi if whenever $\varphi \rightarrow \psi$ is provable in that calculus and $\varphi \in \Gamma$, also $\psi \in \Gamma$. We now demonstrate the proof-theoretic adequacy of these translations.

Lemma 5. For $n$ an odd natural number, let $\Gamma$ be a prime set of formulae closed under either $L \mathrm{E}_{\mathrm{fde} 2}^{\llbracket n \rrbracket}$ or $L \mathrm{E}_{\mathrm{fde} 2}^{\llbracket 2 n \rrbracket}$. Then $\varphi \in \Gamma$ if and only if $\varphi^{N F_{2 n}} \in \Gamma$

Proof. For a zeroth-degree formula $\varphi$, call the depth of $\varphi$ the deepest nesting of the binary connectives within $\varphi$. For example, $\sim_{18} p$ has depth $0, \sim_{3}(p \wedge$ $\left.\left(\sim_{5} q \vee r\right)\right)$ has depth 2 , and so forth. We prove by induction on depth of formulae that for all formulae $\varphi$, the first-degree formulae $\varphi \rightarrow(\varphi)^{N F_{2 n}}$ and $(\varphi)^{N F_{2 n}} \rightarrow \varphi$ are theorems of $L \mathrm{E}_{\mathrm{fde} 2}^{\llbracket n \rrbracket}$ as well as $L \mathrm{E}_{\mathrm{fde} 2}^{\llbracket 2 n \rrbracket}$.

As a basis step, when $\varphi$ has depth $0, \varphi$ is an atomic formula $p$ following a string of $j$ many negation symbols. Hence, when $j<2 n,\left(\sim_{j} p\right)^{N F_{2 n}}=\sim_{j} p$, and the axiom $\sim_{j} p \vdash \sim_{j} p$ witnesses that the two are interderivable. When $j \geq 2 n$, then for some $i$ and $k<2 n, j=(i \cdot 2 n)+k$. So starting with the axiom $\sim_{k} p \vdash \sim_{k} p$ and applying $i$ many instances of either $\left[\sim_{2 n} L\right]$ or $\left[\sim_{2 n} R\right]$ will end in proofs of $\sim_{k} p \rightarrow\left(\sim_{j} p\right)^{N F_{2 n}}$ or $\left(\sim_{j} p\right)^{N F_{2 n}} \rightarrow \sim_{j} p$, respectively.

As induction hypothesis for a formula $\varphi$, assume all formulae $\psi$ whose depth is less than that the depth of $\varphi$, we have proofs of both $\psi \rightarrow(\psi)^{N F_{2 n}}$ and $(\psi)^{N F_{2 n}} \rightarrow \psi$. (Recall that $\psi$ and $\sim_{k} \psi$ are of equal depth for any $k$.)

If $\varphi=\sim_{j}(\xi \wedge \zeta)$ or $\varphi=\sim_{j}(\xi \vee \zeta)$, then the induction hypothesis entails the existence of proofs showing that both $\sim_{j} \xi$ and $\sim_{j} \zeta$ (and, indeed, $\sim_{k} \xi$ and $\sim_{k} \zeta$ for any $k$ ) are interderivable with their respective normal forms.

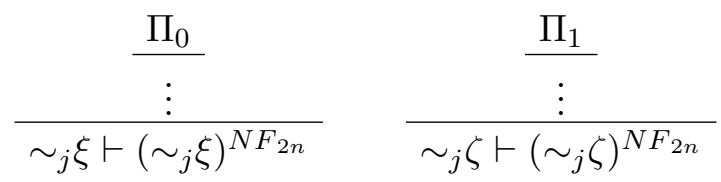

Now, for every sequent in $\Pi_{0}$ construct a new proof $\Pi_{0}^{\prime}$ by placing $\sim_{j} \zeta$ in the rightmost element of every antecedent. Likewise, construct $\Pi_{1}^{\prime}$ by placing $\sim_{j} \xi$ to the leftmost position of every antecedent in $\Pi_{1}$. From these, we obtain the following proof: 


$$
\frac{\frac{\Pi_{0}^{\prime}}{\vdots}}{\frac{\frac{\Pi_{1}^{\prime}}{\sim_{j} \xi, \sim_{j} \zeta \vdash\left(\sim_{j} \xi\right)^{N F_{2 n}}}}{\frac{\sim_{j} \xi, \sim_{j} \zeta \vdash\left(\sim_{j} \zeta\right)^{N F_{2 n}}}{\sim_{j} \xi, \sim_{j} \zeta \vdash\left(\sim_{j} \xi\right)^{N F_{2 n}} \wedge\left(\sim_{j} \zeta\right)^{N F_{2 n}}}}\left[\wedge_{\mathrm{e}} L\right]}\left[\wedge_{\mathrm{e}} R\right]
$$

Because $\left(\sim_{j}(\xi \wedge \zeta)\right)^{N F_{2 n}}$ is defined as $\left(\sim_{j} \xi\right)^{N F_{2 n}} \wedge\left(\sim_{j} \zeta\right)^{N F_{2 n}}$, this establishes that $\varphi \rightarrow(\varphi)^{N F_{2 n}}$ is a theorem of both $L \mathrm{E}_{\text {fde2 }}^{\llbracket n \rrbracket}$ and $L \mathrm{E}_{\text {fde2 } 2}^{\llbracket 2 n \rrbracket}$.

To show that the converse is, too, a theorem, take proofs $\Xi_{0}^{\prime}$ and $\Xi_{1}^{\prime}$ such that:

$$
\frac{\frac{\Xi_{0}^{\prime}}{\vdots}}{\frac{\Xi_{1}^{\prime}}{\left(\sim_{j} \xi\right)^{N F_{2 n}},\left(\sim_{j} \xi\right)^{N F_{2 n}} \vdash \sim_{j} \xi}} \quad \frac{\frac{\Xi^{\prime}}{\left(\sim_{j} \xi\right)^{N F_{2 n}},\left(\sim_{j} \xi\right)^{N F_{2 n}} \vdash \sim_{j} \zeta}}{}
$$

On the guarantee that these proofs exist, we may construct the following proof:

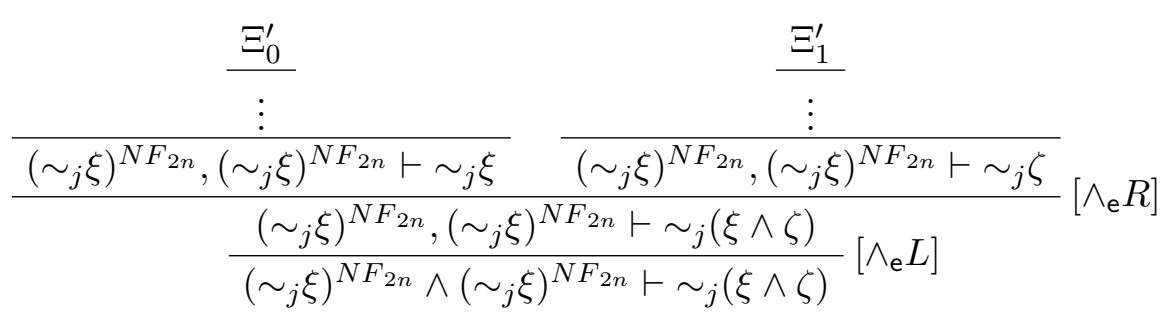

For similar reasons, this proof establishes the theoremhood of $(\varphi)^{N F_{2 n}} \rightarrow \varphi$ in both systems. This covers the case in which $j$ is even. The steps in the case in which $j$ is odd follow from an identical method, as do the cases of even and odd disjunctions, for which reason these details are left to the reader.

Once the induction on depth of formulae is complete, we note that if both $\varphi \rightarrow(\varphi)^{N F_{2 n}}$ and $(\varphi)^{N F_{2 n}} \rightarrow \varphi$ are provable and $\Gamma$ is closed under consequence of $L \mathrm{E}_{\text {fde2 }}^{\llbracket n \rrbracket}$ or $L \mathrm{E}_{\text {fde2 }}^{\llbracket 2 n \rrbracket}$, it follows that $\varphi \in \Gamma$ if and only if $(\varphi)^{N F_{2 n}} \in \Gamma$.

At this point, we have secured the material necessary for a proof of the completeness of any consecution calculus $L \mathrm{E}_{\text {fde } 2}^{\llbracket n \rrbracket}$ with respect to the class of cyclical first-degree models with period $n$.

To begin, we construct the canonical model for any prime set of zerothdegree formulae $\Gamma$ deductively closed under a calculus $L \mathrm{E}_{\mathrm{fde} 2}^{\llbracket n \rrbracket}$. In Routley 
and Routley's canonical model in [6], for any set-up $H$, the set-up $H^{M}$ (i.e., $\left.H^{*}\right)$ is interpreted as the set $\{p \mid \sim p \notin H\}$. We follow a more general-but similar-line, as described in the following definition:

Definition 11. For a prime and non-trivial set of zeroth-degree formulae $\Gamma$ closed under $L \mathrm{E}_{\mathrm{fde} 2}^{\llbracket n \rrbracket}$ consequence, the canonical model of period $n$ is a cyclical model of period $n \mathfrak{M}[\Gamma, n]=\left\langle G, K,{ }^{M}\right\rangle$ interpreting its set-ups $K=$ $\left\{G, H, . ., H^{M_{n-1}}\right\}$ so that $G^{M}=G, H^{M_{n}}=H, G=\varnothing$, and

$$
\text { for all } k \text { such that } 0 \leq k \leq n-1, H^{M_{k}}=\left\{\begin{array}{ll}
\left\{p \mid \sim_{k} p \in \Gamma\right\} & \text { for } k \text { even } \\
\left\{p \mid \sim_{k} p \notin \Gamma\right\} & \text { for } k \text { odd }
\end{array}\right. \text {. }
$$

For example, the atomic set-up $H$ (i.e., $H^{M_{0}}$ ) is just the set of all atoms $p$ appearing in $\Gamma, H^{M}$ is the set of all atoms $p$ whose negation $\sim p$ does not appear in $\Gamma, H^{M M}$ is the set of all atoms $p$ such that $\sim \sim p \in \Gamma$, and so forth.

That the definition of a canonical model correctly interprets each of its set-ups can be established as follows:

Lemma 6. Let $\Gamma$ be prime and deductively closed under $L \mathrm{E}_{\mathrm{fde} 2}^{\llbracket n \rrbracket}$. Then for any $\varphi$ a zeroth-degree formula, $\varphi \in \Gamma$ if and only if $\mathfrak{M}[\Gamma, n], H \Vdash \varphi$.

Proof. By Lemmas 4 and 5, we may assume without loss of generality that $\varphi$ is in normal form. Then we prove the present lemma by induction on complexity of $\varphi$. As a basis step, the construction ensures that $p \in H$ if and only if $p \in \Gamma$.

For induction hypothesis, suppose that this property holds for all subformulae of $\varphi$. Then we must consider the following three cases:

- Suppose $\varphi$ is an atom $p$ following a sequence of $k$ negation signs. Now, let $j=k \bmod n$ (i.e., let $j$ be the modulus of $k \bmod n$ ). Then $\sim_{k} p \in$ $\Gamma$ iff $\sim_{j} p \in \Gamma$. If $j$ is even, then by construction of the canonical model, we know that $H^{M_{j}}=\left\{p \mid \sim_{j} p \in \Gamma\right\}$. Hence, in this case $\sim_{j} p \in \Gamma$ is equivalent to $p \in H^{M_{j}}$, which is itself equivalent to $p \in H^{M_{k}}$ and, in turn, equivalent to $H \Vdash \sim_{k} p$. If $j$ is odd, then similarly, because $H^{M_{j}}=\left\{p \mid \sim_{j} p \notin \Gamma\right\}, \sim_{j} p \in \Gamma$ is equivalent to $p \notin H^{M_{j}}$, which is, in turn, equivalent to $p \notin H^{M_{k}}$ and $H \Vdash \sim_{k} p$.

- In the case of conjunction, $\varphi \wedge \psi \in \Gamma$ iff $\varphi \in \Gamma$ and $\psi \in \Gamma$. By induction hypothesis, this is equivalent to both $H \Vdash \varphi$ and $H \Vdash \psi$. This is precisely the truth condition for $H \Vdash \varphi \wedge \psi$. 
- For disjunction, by primeness of $\Gamma, \varphi \vee \psi \in \Gamma$ iff $\varphi \in \Gamma$ or $\psi \in \Gamma$. An appeal to the induction hypothesis and the truth condition for disjunction secures the equivalence with $H \Vdash \varphi \vee \psi$.

Having shown the adequacy of each canonical model $\mathfrak{M}[\Gamma, n]$, we are now prepared to prove the completeness of each of the systems described in the foregoing pages with respect to an appropriate class of set-up models with cyclical mate functions:

Theorem 1 (Completeness of the Consecution Calculi). For a natural number $n$, if $\varphi \rightarrow \psi$ is not derivable in $L \mathrm{E}_{\mathrm{fde} 2}^{\llbracket n \rrbracket}$ then there exists some cyclical model $\mathfrak{M}$ of period $n$ such that $\mathfrak{M}, G \nVdash \varphi \rightarrow \psi$.

Proof. Suppose that $\varphi \rightarrow \psi$ is not a theorem. Then let $\Gamma$ be the prime, deductively closed set whose existence is promised by Lemma 3 in such that $\varphi \in \Gamma$ although $\psi \notin \Gamma$. By Lemma 6 , the proof-theoretic facts that $\varphi \in \Gamma$ and $\psi \notin \Gamma$ are reflected semantically in the canonical model $\mathfrak{M}[\Gamma, n]$ by the fact that $\mathfrak{M}[\Gamma, n], H \Vdash \varphi$ while $\mathfrak{M}[\Gamma, n], H \not \models \psi$. But this $H$ is sufficient to ensure that $\mathfrak{M}[\Gamma, n], G \nVdash \varphi \rightarrow \psi$, as required.

The corresponding property attesting to the soundness of appropriate model theories is far easier to establish.

Theorem 2 (Soundness of the Consecution Calculi). For a natural number $n$, if $\varphi \rightarrow \psi$ is provable in $L \mathrm{E}_{\text {fde2 }}^{\llbracket n \rrbracket}$ then for all cyclical models $\mathfrak{M}$ of period $n$ for which $\mathfrak{M}, G \Vdash \varphi$, also $\mathfrak{M}, G \Vdash \psi$.

Proof. It is easy to confirm that for each axiom of any system $L \mathrm{E}_{\mathrm{fde} 2}^{\llbracket n \rrbracket}$, whenever all members of the antecedent are true at a set-up $H$ in a model with period $n$ then some member of the succedent is true at that set-up. For instances of [Axiom], there exists some formula $\varphi$ appearing in both antecedent and succedent of the axiom. Trivially, then, whenever $H$ makes true all elements of the antecedent, it a fortiori makes true $\varphi$, establishing that some element of the succedent $(i . e ., \varphi)$ is true at $H$. When $n$ is odd, the axioms $\left[\perp_{n} L\right]$ and $\left[\top_{n} R\right]$ are likewise adequate. Because in models in which ${ }^{M}$ has period $n$ we know that $H^{M_{n}}=H$, it is impossible that both $H \Vdash \varphi$ and $H \Vdash \sim_{n} \varphi$ hold insofar as the latter entails that $H \nVdash \varphi$. Likewise, either $H \Vdash \varphi$ or $H \Vdash \sim_{n} \varphi$ must hold, as this amounts to saying that either $H \Vdash \varphi$ or $H \nVdash \varphi$. 
Now, for any rule of a system $L \mathrm{E}_{\text {fde2 } 2}^{\llbracket n \rrbracket}$ licensing the inference of a sequent $\Gamma \vdash \Delta$ from one or two top sequents $\Gamma^{\prime} \vdash \Delta^{\prime}$ (and possibly $\Gamma^{\prime \prime} \vdash \Delta^{\prime \prime}$ ), we can confirm that whenever all set-ups making true each formula in $\Gamma^{\prime}$ make true some formula in $\Delta^{\prime}$ (and, if applicable, mutatis mutandis for $\Gamma^{\prime \prime} \vdash \Delta^{\prime \prime}$ ), it also holds that a set-up making true every formula in the antecedent $\Gamma$ must make true some formula in the succedent $\Delta$.

To confirm that this property holds of each rule is straightforward. For negation, when $n$ is odd, in models with period $n, H \Vdash \varphi$ precisely when $H^{M_{2 n}} \Vdash \varphi$, verifying the adequacy of $\left[\sim_{2 n} L\right]$ and $\left[\sim_{2 n} R\right]$. When $n$ is even, $H \Vdash \varphi$ precisely when $H^{M_{n}} \Vdash \varphi$, attesting to the adequacy of $\left[\sim_{n} L\right]$ and $\left[\sim_{n} R\right]$ with respect to these models. In, e.g., the case of $\left[\wedge_{\mathrm{e}} L\right]$, the details of Lemma 4 reveal that $H$ makes true $\sim_{k} \varphi$ and $\sim_{k} \psi$ precisely when $H$ makes true $\sim_{k}(\varphi \wedge \psi)$ whenever $k$ is even. Indeed, the adequacy of the even and odd rules for conjunction and disjunction may be recognized by the reader without difficulty by a cursory glance at the details Lemma 4 . Hence, the task of explicitly confirming the adequacy of each rule is set aside.

With these systems characterized to a reasonable degree and the bridge between the model theory and proof theory having been shown to be adequate, we now proceed to examine some additional metalogical properties that are especially interesting in the context of cyclical first-degree models.

\section{Parity of Cyclical Mate Functions and Metalog- ical Properties}

At several points in the previous sections, we have encountered the feature that the parity of the period of a mate function-i.e., whether the period of ${ }^{M}$ is even or odd-leads to a dramatic difference between the consequence relations corresponding to the even-parity systems and those corresponding to odd-parity systems. Upon reflection from the perspective of the set-up semantics, the semantic feature that, arguably, is most responsible for the pronounced distinction lies in the capacity of the odd-parity systems for expressing properly exclusionary notions of truth and falsity that are local to the same set-up $H$.

In Sylvan and Plumwood's set-up semantics with the Routley star, from a set-up $H$ it is not in general possible to describe or express what is false at $H$. When the period of the operator is even - as in the case of the semantics for first-degree entailment- $H$ is incapable of reaching itself in anything other than an even number of applications of $*^{*}$. On the other 
hand, $H$ can only speak meaningfully about falsity in situations $H^{*_{n}}$ where $n$ is odd, ensuring that there exists no method of defining and operator • such that $H \Vdash \bullet \varphi$ if and only if $H \nVdash \varphi$. Both even-parity and odd-parity systems - through a lengthy iteration of an appropriate (and even) string of negations - enable a set-up $H$ to trace its mate function back to itself to indirectly assert what is true at $H$. In contrast, only the odd-parity systems enable one to start at $H$ and trace the mate function in order to express what is false at $H$.

As suggested in Section 1, the divide between even and odd parity serves as a generalization of the properties of the Routley star that correspond alternately to classical logic or to first-degree entailment. With our net cast more widely to this family of periodic mate functions, the foregoing discussion licenses us to recast a number of distinctions between classical logic and first-degree entailment as not merely a matter of involutivity but as a matter of parity. In this spirit, we will survey connections between several further metalogical properties of deductive systems and investigate how they correlate to the distinction between even and odd parity of the mate function.

\subsection{Relevance and Parity}

It is well-known that first-degree entailment is a member of the family of relevance or relevant logics, a family sometimes motivated by the thesis that successful arguments presuppose some degree of relevance between the premises and the conclusion. Authoritative accounts of the motivations and philosophy for relevant logics can be found in the volumes [5] and [1]. In a formal setting of, e.g., propositional logic, this intuitive feature is often identified with the requirement that for all theorems $\varphi \rightarrow \psi$, there exists some atomic formula $p$ appearing in both $\varphi$ and $\psi$.

This criterion - the variable sharing property - is definable in the following way:

Definition 12. A first-degree logic has the variable sharing property (VSP) if a first degree formula $\varphi \rightarrow \psi$ is a theorem only if there exists an atom $p$ appearing in both $\varphi$ and $\psi$.

It is frequently noted that first-degree entailment enjoys the VSP, making it reasonable to inquire about the fate of this property - and of related properties - in the case of our calculi $L \mathrm{E}_{\text {fde2 }}^{\llbracket n \rrbracket}$.

There are, as it turns out, some intriguing connections between the calculi described in this paper and the VSP. We are, for example, able to 
describe and impose refinements of the VSP by asserting stronger criteria and to associate these refined relevance properties with the calculi in our family for which they hold. Interestingly, the parity of a cycle $n$ plays an important role in determining which - if any - flavors of the VSP hold of a calculus $L \mathrm{E}_{\mathrm{fde} 2}^{\llbracket 2 \rrbracket}$.

Initially, emphasizing the cyclical nature of Routley stars qua mate functions enables us to describe a stronger form of the property. Interestingly, we are capable of demonstrating that first-degree entailment enjoys a stronger version of the VSP than is often acknowledged. Consider the following definition:

Definition 13. A first-degree logic has the cyclical variable sharing property with modulus $n$ (VSP $\bmod n$ ) if a first degree formula $\varphi \rightarrow \psi$ is a theorem only if there exists an atom $p$ and natural numbers $j$ and $k$ such that $j \equiv k$ $\bmod n$ for which $p$ appears within the scope of $j$ negation signs in $\varphi$ and $k$ negation signs in $\psi$.

Clearly, the VSP $\bmod n$ counts the VSP as a special case, albeit one in which the number of negation signs within which a parameter falls is irrelevant. This allows us to reframe a result due to Anderson and Belnap in [1, p. 253] that first-degree entailment enjoys not only the VSP simpliciter but also the stronger property of the VSP $\bmod 23^{3}$

Observation 3. First-degree entailment enjoys the VSP $\bmod 2$.

Proof. We prove this by contradiction by assuming that first-degree entailment does not enjoy the VSP mod 2.

By the definition of normal form, for a zeroth-degree formula $\varphi$, the syntactic form of its translation $(\varphi)^{N F_{2 n}}$ is the result of the repeated application of disjunctions and conjunctions to a set of literals (i.e., formulae that are either atoms $p$ or negated atoms $\sim p$ ).

Say that an instance of an atom $p$ appears positively in a formula $\varphi$ if it is within the scope of an even number of negation signs and that it appears negatively if it is within the scope of an odd number of negation signs. Now, suppose that there exists no atom $p$ such that $p$ appears either positively in both $\varphi$ and $\psi$ or negatively in both $\varphi$ and $\psi$. Then by following the definition for $(\varphi)^{N F_{2}}$ and $(\psi)^{N F_{2}}$, each $p$ appearing positively (respectively, negatively) in $\varphi$ appears positively (respectively, negatively) in $(\varphi)^{N F_{2}}$, and mutatis mutandis for $\psi$.

\footnotetext{
${ }^{3}$ I am grateful for an anonymous referee's pointing out to me that the theorem described in [1, p. 253] is a proof of the VSP mod 2 with different terminology.
} 
Now, consider some $L \mathrm{E}_{\text {fde2 } 2}^{\llbracket 2 \rrbracket}$ proof $\Pi$ of the theorem $(\varphi)^{N F_{2}} \rightarrow(\psi)^{N F_{2}}$. We consider a method of modifying $\Pi$ that yields a new proof $\Pi^{\prime}$ by replacing each instance of a literal $\sim p$ with a new atomic formula $\hat{p}$ and call the formulae resulting from this replacement $(\hat{\varphi})^{N F_{2}}$ and $(\hat{\psi})^{N F_{2}}$. Now, because $(\varphi)^{N F_{2}}$ and $(\psi)^{N F_{2}}$ are in normal form, there will be no instances in which new negation symbols are introduced or commuted through a disjunction or conjunction. We may, in other words, treat each negated literal $\sim p$ as a unit, whose interpretation is wholly independent from that of the formula $p$.

Hence, in $\Pi^{\prime}$ we have an $L \mathrm{E}_{\text {fde2 }}^{\llbracket 2 \rrbracket}$ proof of the theorem $(\hat{\varphi})^{N F_{2}} \rightarrow(\hat{\psi})^{N F_{2}}$. But because $\varphi$ and $\psi$ were assumed to have neither a positive atom nor a negative atom in common, the nature of our replacement entails that $(\hat{\varphi})^{N F_{2}}$ and $(\hat{\psi})^{N F_{2}}$ have no atomic formulae at all in common. But this conclusion contradicts the well-known fact that first-degree entailment enjoys the VSP simpliciter. We conclude that first-degree entailment indeed enjoys the stronger property of the VSP mod 2.

Having established that the well-known logic of first-degree entailment enjoys the VSP mod 2, we return to the general case and examine the connection between the cyclical variable sharing properties and the logics with periodic mate functions.

The connection between the two is very tight indeed, with the period of a system $L \mathrm{E}_{\mathrm{fde} 2}^{\llbracket 2 n \rrbracket}$ corresponding to the period of the particular species of the VSP it enjoys.

Observation 4. For a system with periodicity $2 n$, the system enjoys the $V S P \bmod 2 n$.

Proof. Suppose for contradiction that no variable appears in both $\varphi$ and $\psi$ within the scope of $j$ and $k$ many negation signs, respectively, where $j \equiv k$ $\bmod 2 n$. Furthermore, let $\Pi$ be an $L \mathrm{E}_{\text {fde2 }}^{\llbracket 2 n \rrbracket}$ proof of $\varphi \rightarrow \psi$.

As we have established, $\varphi \vdash \psi$ is derivable in $L \mathrm{E}_{\mathrm{fde} 2}^{\llbracket 2 n \rrbracket}$ if and only if $(\varphi)^{N F_{2 n}} \vdash(\psi)^{N F_{2 n}}$ is derivable. Hence, we can find a proof $\Pi^{\prime}$ of $(\varphi)^{N F_{2 n}} \vdash$ $(\psi)^{N F_{2 n}}$ for which every application of a rule is a simple introduction of either conjunction or disjunction.

Take every leaf of $\Pi^{\prime}$ and replace each appearance of a formula $\sim_{k} p_{j}$ with a new atom $p_{\pi(j, k)}$, where $\pi$ is a pairing function. Now, construct a proof $\Pi^{\prime \prime}$ by applying precisely the same rules of $\Pi^{\prime}$ to these leafs. Because there are no applications of rules $\left[\sim_{2 n} L\right]$ or $\left[\sim_{2 n} R\right]$, every rule applied in $\Pi^{\prime}$ is a fortiori a correct rule of $L \mathrm{E}_{\mathrm{fde} 2}^{\llbracket 2 \rrbracket}$. Hence, $\Pi^{\prime \prime}$ is also an $L \mathrm{E}_{\mathrm{fde} 2}^{\llbracket 2 n \rrbracket}$ proof 
of a sequent $(\hat{\varphi})^{N F_{2 n}} \vdash(\hat{\psi})^{N F_{2 n}}$ in which there are no atomic formulae in common between $(\hat{\varphi})^{N F_{2 n}} \vdash(\hat{\psi})^{N F_{2 n}}$. But this contradicts the fact that first-degree entailment enjoys the VSP simpliciter. We thus conclude that $L \mathrm{E}_{\text {fde2 }}^{\llbracket 2 n \rrbracket}$ enjoys the VSP $\bmod 2 n$.

Now, we have covered the even cases and seen that the value of the period of a model's mate function corresponds exactly to the period of the flavor of the VSP enjoyed by one of these systems. In the particular case of firstdegree entailment, we have also been able to attribute to the system a more refined property (i.e., the VSP mod 2) than the coarser VSP.

We then inquire after the relationship between relevance and the systems in which the mate function has odd periodicity. To begin, recall that the systems $L \mathrm{E}_{\text {fde2 }}^{\llbracket n \rrbracket}$ for odd $n$ have axioms $\left[\perp_{n} L\right]$ and $\left[\top_{n} R\right]$ that are essentially generalizations of ex contradictione quodlibet and tertium non datur. Just as the more familiar versions of these principles are notorious for the introduction of irrelevance, the generalized axioms, too, have the potential to introduce irrelevance into a proof. Consequently, we find that the systems with odd parity uniformly fail to satisfy any species of the variable sharing property.

Observation 5. For a system with odd periodicity $2 n+1$, for no $m$ does the system enjoy the VSP mod $m$.

Proof. We produce a counterexample replicable in any system $L \mathrm{E}_{\mathrm{fde} 2}^{\llbracket 2 n+1 \rrbracket}$ :

$$
\frac{\overline{p, \sim_{2 n+1} p \vdash q}}{p \wedge \sim_{2 n+1} p \vdash q}[\text { Axiom] }
$$

The theoremhood of $\left(p \wedge \sim_{2 n+1} p\right) \rightarrow q$ clearly violates the VSP simpliciter and, consequently, violates the VSP $\bmod m$ for any $m$.

It appears that the ability to speak at $H$ about what is false at $H$-whether directly (as in classical logic) or indirectly via a lengthy chain of iterated negation symbols - is a talent peculiar to the calculi with odd parity. Implicit in the additional expressivity odd parity grants us-i.e., implicit in the capacity to not merely assert that a negation is true at a set-up $H$, but also to deny that a formula is true - is the capacity to exclude the truth of a formula in a manner not available to, e.g., first-degree entailment.

In the following joint corollary, Observations 4 and 5 provide a compelling illustration of the severity of the logical division that follows in the wake of differences in parity. 
Corollary 1. For a consecution calculus $L \mathrm{E}_{\mathrm{fde} 2}^{\llbracket n \rrbracket}$, the system is a relevant logic if and only if $n$ is even.

With this corollary in mind, that classical logic (i.e., set-up models whose mate function has period 1) is not a relevant logic while first-degree entailment (i.e., set-up models with period 2) is revealed to be merely a single facet of a broader phenomenon.

We have surveyed one of the hallmark properties of relevant logics, given it a refinement, and discovered that the extent of these properties is surprisingly in alignment with the distinction between even and odd parity of a mate function. We now change gears in our consideration of the relationship between parity of a mate function and metalogical properties, exchanging themes from the tradition of relevant logic for those of a different school in the field of non-classical logic.

\subsection{Formal Inconsistency and Parity}

We now look to a tradition in non-classical logic that is largely independent of the relevant logic tradition, namely, the field of logics of formal inconsistency (LFIs). During this section, we will examine the general relationships that hold between instances of our calculi and the hallmark features of LFIs, which are thoroughly documented in the handbook article [2]. Intriguingly, for many of the central properties described by proponents of LFIs, we again find that whether a system enjoys these properties in large part mirrors the division of our systems by parity. In contrast to the hallmark property of the VSP, which we found to be coextensional with the even-parity calculi, in the LFI case, we will discover that key properties from the LFI camp-like gentle explosiveness - hold only of the odd-parity calculi.

The notion of paraconsistency plays a key role in the sequel, so it is worth briefly reviewing the notion. A deductive system with a negation is referred to as paraconsistent if ex contradictione quodlibet fails, i.e., when there are cases in which a contradiction does not license one to conclude to arbitrary formulae.

Definition 14. A system with a consequence relation $\vdash$ and negation symbol $\sim$ is paraconsistent when there exists a set of formulae $\Gamma$ and formulae $\varphi$ and $\psi$ such that:

- $\Gamma, \varphi, \sim \varphi \nvdash \psi$

Now, logics of formal inconsistency, like relevant logics, are instances of paraconsistent logics, although the two schools disagree with respect to 
their organizing principles. LFIs are largely motivated by the thesis that even if in certain, abnormal contexts one might tolerate contradictions, there exist situations demanding that we retain sufficient control — and sufficient expressivity - to exclude the appearance of contradictions in a theory. While, for example, the assertion that the Liar is both true and false may be tolerable in the context of philosophical debate, the same cannot be said of sensor readings on an airplane during flight. The additional machinery developed by the LFI community permits the identification of "normal" cases in which contradictions are intolerable, thereby distinguishing them from the "abnormal" cases in which contradictions may be countenanced.

This additional control in appropriate contexts is captured by the property of a system's being gently explosive, by which the principle of explosion can be deployed in particular, controlled contexts.

Definition 15. For a deductive system with consequence relation $\vdash$ and negation $\sim$, let $\bigcirc(p)$ be a (possibly empty) set of formulae depending only on the value of $p$ such that there exist formulae $\varphi$ and $\psi$ for which:

- $\bigcirc(\varphi), \varphi \nvdash \psi$

- $\bigcirc(\varphi), \sim \varphi \nvdash \psi$

Then the system is gently explosive if for all sets of formulae $\Gamma$ and formulae $\varphi$ and $\psi$ :

- $\Gamma, \bigcirc(\varphi), \varphi, \sim \varphi \vdash \psi$

This set $\bigcirc(\varphi)$-often represented by a unary "consistency connective" oprovides a syntactic mark signaling that a formula $\varphi$ is to be treated "classically" and indicating that the presence of both $\varphi$ and $\sim \varphi$ in a theory should be "explosive."

Putting these pieces together, then, leads to a natural definition for a logic of formal inconsistency, in which the dual desiderata of maintaining paraconsistency in general and the ability to deploy explosion in special cases are reflected:

Definition 16. A logic of formal inconsistency with respect to a negation $\sim$ is a deductive system that is both paraconsistent and gently explosive. 
Now let us investigate the relationship that holds between the key properties of LFIs and the deductive systems defined in this paper. We begin by examining the extent of these properties piecemeal.

First, we may observe that almost all of the consecution calculi introduced in the foregoing material correspond to paraconsistent logics:

Observation 6. For all $n \geqslant 1$, the consequence relation corresponding to the consecution calculus $L \mathrm{E}_{\mathrm{fde} 2}^{\llbracket n \rrbracket}$ is paraconsistent.

Proof. For any of our consecution calculi $L \mathrm{E}_{\mathrm{fde} 2}^{\llbracket n \rrbracket}$, the definition of paraconsistency entails that the failure of the property is equivalent to the provability of all sequents in which the antecedent includes as formulae $\varphi$ and $\sim \varphi$. As a special case, the failure of paraconsistency requires the provability of the sequent $p \wedge \sim p \vdash q$. However, the rules described for our calculi permit only one possible proof of this sequent:

$$
\overline{p, \sim p \vdash q}\left[\perp_{1} L\right]
$$

Note, however, that this proof necessarily requires the use of axiom $\left[\perp_{1} L\right]$ and that this rule is available only to the system $L \mathrm{E}_{\text {fde2 }}^{\llbracket 1 \rrbracket}$. Consequently, this proof cannot be reproduced in any calculus $L \mathrm{E}_{\mathrm{fde} 2}^{\llbracket n \rrbracket}$ for which $n \neq 1$, allowing us to conclude the paraconsistency of each consecution calculus in which $n \neq 1$.

Likewise, we find that the gently explosive systems in our framework are exactly those logics characterized by models whose mate function has an odd period.

Observation 7 . The consequence relation corresponding to a calculus $L \mathrm{E}_{\mathrm{fde} 2}^{\llbracket n \rrbracket}$ is gently explosive if and only if $n$ is odd.

Proof. We consider the two cases in which $n$ is either odd or even. For odd $n \geq 1$, we provide a model-theoretic argument that $L \mathrm{E}_{\text {fde } 2}^{\llbracket n \rrbracket}$ is gently explosive by defining $\bigcirc(p)$ as the formula $\sim_{n}(p \wedge \sim p)$. Because $H^{M_{n}}=H$, whenever $H \Vdash \bigcirc(\varphi)$ holds in some model, either $H \nVdash \varphi$ or $H \nVdash \sim \varphi$ must follow. Hence, while the formulae $\bigcirc(p)$ and $p$ are jointly satisfiable - and the formulae $\bigcirc(p)$ and $\sim p$ are jointly satisfiable - for no formula $\varphi$ is there a model with odd periodicity $n$ in which $\bigcirc(\varphi), \varphi$, and $\sim \varphi$ are jointly satisfied at a set-up. Hence, for any odd $n$ - to include the case in which $n=1$ - the calculus $L \mathrm{E}_{\mathrm{fde} 2}^{\llbracket n \rrbracket}$ is gently explosive.

Finally, we consider even natural numbers $n$. To begin, recall Observation 4 , in which we determined that for all even natural numbers $n, L \mathrm{E}_{\mathrm{fde} 2}^{\llbracket n \rrbracket}$ 
enjoys the variable-sharing property. Were such a system to be gently explosive, for any atomic formula $p$, we can expand the set of atoms to include a new atom $\hat{q}$ not appearing in $\bigcirc(p)$ for which $\hat{q} \neq p$, and prove the sequent $\bigcirc(p), p, \sim p \vdash \hat{q}$. But there are no variables in common between the antecedent and succedent of this sequent, whence the provability of this sequent is ruled out by the VSP. Consequently, for any even $n$, the relevance properties exhibited by $L \mathrm{E}_{\text {fde2 }}^{\llbracket n \rrbracket}$ prevent it from being gently explosive.

Consequently, it is precisely when $n$ is odd that $L \mathrm{E}_{\mathrm{fde} 2}^{\llbracket n \rrbracket}$ is gently explosive.

We may apply Observations 6 and 7 to immediately yield the following characterization of the conditions under which one of our calculi is an LFI:

Corollary 2. The logic corresponding to a consecution calculus $L \mathrm{E}_{\mathrm{fde} 2}^{\llbracket n \rrbracket}$ is a logic of formal inconsistency with respect to $\sim$ if and only if $n$ is an odd number greater than 1.

There remain a number of even finer assertions that we could make concerning the relationship between the hallmark properties of LFIs and the present systems with cyclical mate functions. For example, recall that the proof of Observation 7 provided a definable consistency operator for each system $L \mathrm{E}_{\text {fde } 2}^{\llbracket n \rrbracket}$ where $n$ is odd and $n>1$. Hence, these systems qua LFIs enjoy the additional label of $d C$-system, that is, an LFI for which a single formula that is definable in a system's language plays the role of the set $\bigcirc(p)$.

It is apparent from this section that the generality gained by considering Sylvan's mate function reveals connections between set-up models and logics of formal inconsistency that are suppressed when the stronger Routley star is employed. We leave further investigation of these connections for future work.

\section{Conclusions}

During this discussion, we have examined the behavior of Richard Sylvan's mate function - a weaker version of the Routley star - and explored the consequences of evaluating negation in first-degree models in this way. Distinguishing these models on the basis of the period of the mate function determined a countably infinite collection of distinct first-degree logics, and we reviewed soundness and completeness proofs providing each such logic with an adequate model theory and a correct proof theory. Moreover, we gave 
an exhaustive characterizaton of the conditions under which one of these systems is a subsystem of another.

Along the way, we observed that the properties of these systems naturally unpacked themselves into two very distinct partitions and that this divisions was tightly correlated with the parity of mate functions, so that the logics in which mate functions have even parity differ dramatically from those systems whose mate functions have odd parity. Upon further examination, we were able to provide more illumination into this divide by demonstrating, for example, that logics corresponding to mate functions of even parity are exactly the systems that are relevant logics while logics corresponding to functions with odd parity are exactly the class of logics that are gently explosive.

I find this division - and how closely it mirrors themes from both relevant and LFI camps - to be extremely intriguing for its own sake. But there ought to be additional merits to studying different constraints on the mate function and the consequence relations that these constraints induce. For example, there is currently increasing interest in first-degree systems that are either properly contained in - or are otherwise very closely related to - the logic of first-degree entailment. The semantical analyses of these systems have followed numerous semantic approaches - ranging from many-valued semantics to situation semantics - but Sylvan and Plumwood's framework has not yet been conscripted in the effort to better understand these systems. This is unfortunate, as our examination of these systems in the contexts of relevant logics and logics of formal inconsistency has demonstrated that looking to the mate function drastically increases the reach and generality of set-up semantics. It seems that underscoring the novelty and flexibility of Sylvan's mate function may serve to prompt researchers to frame their analyses of these weak, first-degree systems in a novel way.

\section{Acknowledgement}

I am grateful for the extraordinarily helpful remarks of an anonymous referee. 


\section{References}

[1] A. R. Anderson and N. D. Belnap, Jr., Entailment: The logic of relevance and necessity, vol. I, Princeton University Press, Princeton, 1975.

[2] W. Carnielli, M. E. Coniglio, and J. Marcos, Logics of formal inconsistency, Handbook of Philosophical Logic (D. Gabbay and F. Guenthner, eds.), vol. 14, Springer, Netherlands, 2007, pp. 15-107.

[3] G. Robles, A note on the non-involutive Routley star, Bulletin of the Section of Logic 37 (2008), no. 1, 19-27.

[4] , The non-involutive Routley star: Relevant logics without weak double negation, Teorema 29 (2010), no. 3, 103-116.

[5] R. Routley, R. K. Meyer, V. Plumwood, and R. T. Brady, Relevant logics and their rivals, vol. 1, Ridgeview, Atascadero, CA, 1982.

[6] R. Routley and V. Routley, The semantics of first degree entailment, Noûs 6 (1972), no. 4, 335-359. 